\title{
NASA's Global Precipitation Mission Ground Validation Segment
}

\author{
Mathew R. Schwaller \\ NASA / Goddard Space Flight Center \\ Greenbelt, MD, USA 20771 \\ mathew.r.schwaller@nasa.gov
}

\begin{abstract}
NASA is designing a Ground Validation Segment (GVS) as one of its contributions to the Global Precipitation Measurement (GPM) mission. The GPM GVS provides an independent means for evaluation, diagnosis, and ultimately improvement of the GPM spaceborne measurements and precipitation products. NASA's GPM GVS concept calls for a combination of direct observations executed within a Multidimensional Observing Volume (MOV) and model-based analyses executed by a Satellite Simulator Model (SSM). The MOV consists of ground-based instruments that measure local surface and atmospheric properties required for GPM validation. The SSM utilizes MOV measurements in a forward numerical model. The goal of the SSM forward modeling is calculation of the following properties: top-ofatmosphere microwave radiative quantities to within sensor noise of those measured by the GPM Core Satellite, precipitation quantities identical to those generated by the standard GPM precipitation retrieval algorithms, and quantitative/objective error estimates of both sets of quantities. At present, the GVS is in the early design stage and various scenarios have been generated to assess how it will be used in the GPM era. The GPM GVS will be operational in the year prior to the launch of the GPM core satellite, which has a launch date scheduled for December 2010.
\end{abstract}

\section{INTRODUCTION: GPM AND GROUND VALIDATION}

GPM Ground Validation (GV) supports the overall GPM mission objectives including improvement in predicting terrestrial weather, climate, and hydrometeorology through a better observational understanding of the global water cycle. To help meet these objectives, the GPM GV has the following subsidiary goals:

1. Quantify the uncertainties in GPM standard precipitation retrieval algorithms

2. Contribute to the improvement of GPM precipitation retrieval algorithms throughout the mission by

- validating the radiative transfer models that serve as the basis for the GPM precipitation retrieval algorithms

- validating the assumptions needed to parameterize these models, and
3. Understand the time and space error characteristics of GPM precipitation products generated by these algorithms.

Achieving these goals is seen as a necessary step for improved GPM data products and for increased utilization of these products in Global Climate Models (GCMs), Numerical Weather Prediction (NWP) models, and hydrometeorological models for climate and weather forecasting. An overview of the GPM mission, including the GPM Microwave Imager (GMI) and Dual-frequency Precipitation Radar (DPR) instruments can be found in Flaming (2004).

The conceptual framework for GPM GV is based on the notion of a "multidimensional observing volume" and "satellite simulator model" as briefly described below.

\section{GROUND VALIDATION COMPONENTS}

\section{A. Multidimensional Observing Volume (MOV)}

The MOV consists of ground-based instruments that measure local surface and atmospheric properties required for GPM validation. The specification of MOV instrumentation is still to be determined, but example sensors may include surface precipitation gauges and disdrometers, ground-based radars and radiometers, Doppler profilers, and other meteorological sensors. These instruments will be used in the direct observation or retrieval of parameters that drive the forward model within the SSM (as described below). Specifying the parameters required by the SSM is still to be determined, but the MOV instruments may measure, for example, active and passive microwave (MW) radiation intensities (e.g., radar reflectivity, $\mathrm{Z}$, and radiometer brightness temperature, $\mathrm{Tb}$ ), meteorological quantities (e.g., temperature, pressure, winds), hydrological constituent concentrations (e.g., water vapor, liquid/ice hydrometeor mixing ratios), hydrometeor size spectra (including, for example, liquid droplet and ice particle size distributions), and precipitation rates.

\section{B. Satellite Simulation Model (SSM)}

The SSM utilizes MOV measurements in a forward numerical model, the goal of which is the calculation of: microwave radiative quantities identical to within sensor noise of those measured by the GPM Core Satellite (or other targeted satellites), precipitation quantities identical to those generated by the standard algorithms, and quantitative/objective error 


\section{Report Documentation Page}

Form Approved

OMB No. 0704-0188

Public reporting burden for the collection of information is estimated to average 1 hour per response, including the time for reviewing instructions, searching existing data sources, gathering and maintaining the data needed, and completing and reviewing the collection of information. Send comments regarding this burden estimate or any other aspect of this collection of information,

including suggestions for reducing this burden, to Washington Headquarters Services, Directorate for Information Operations and Reports, 1215 Jefferson Davis Highway, Suite 1204, Arlington

VA 22202-4302. Respondents should be aware that notwithstanding any other provision of law, no person shall be subject to a penalty for failing to comply with a collection of information if it

does not display a currently valid OMB control number.

\begin{tabular}{|c|c|c|}
\hline $\begin{array}{l}\text { 1. REPORT DATE } \\
\mathbf{2 5} \text { JUL } \mathbf{2 0 0 5}\end{array}$ & $\begin{array}{l}\text { 2. REPORT TYPE } \\
\text { N/A }\end{array}$ & $\begin{array}{l}\text { 3. DATES COVERED } \\
\text { - }\end{array}$ \\
\hline \multirow{3}{*}{\multicolumn{2}{|c|}{$\begin{array}{l}\text { 4. TITLE AND SUBTITLE } \\
\text { NASAs Global Precipitation Mission Ground Validation Segment }\end{array}$}} & 5a. CONTRACT NUMBER \\
\hline & & 5b. GRANT NUMBER \\
\hline & & 5c. PROGRAM ELEMENT NUMBER \\
\hline \multirow{3}{*}{\multicolumn{2}{|c|}{ 6. AUTHOR(S) }} & 5d. PROJECT NUMBER \\
\hline & & 5e. TASK NUMBER \\
\hline & & 5f. WORK UNIT NUMBER \\
\hline \multicolumn{2}{|c|}{$\begin{array}{l}\text { 7. PERFORMING ORGANIZATION NAME(S) AND ADDRESS(ES) } \\
\text { NASA / Goddard Space Flight Center Greenbelt, MD, USA } 20771\end{array}$} & $\begin{array}{l}\text { 8. PERFORMING ORGANIZATION } \\
\text { REPORT NUMBER }\end{array}$ \\
\hline \multirow{2}{*}{\multicolumn{2}{|c|}{ 9. SPONSORING/MONITORING AGENCY NAME(S) AND ADDRESS(ES) }} & 10. SPONSOR/MONITOR'S ACRONYM(S) \\
\hline & & $\begin{array}{l}\text { 11. SPONSOR/MONITOR'S REPORT } \\
\text { NUMBER(S) }\end{array}$ \\
\hline
\end{tabular}

12. DISTRIBUTION/AVAILABILITY STATEMENT

Approved for public release, distribution unlimited

13. SUPPLEMENTARY NOTES

See also ADM001850, 2005 IEEE International Geoscience and Remote Sensing Symposium Proceedings (25th) (IGARSS 2005) Held in Seoul, Korea on 25-29 July 2005. , The original document contains color images.

14. ABSTRACT

15. SUBJECT TERMS

16. SECURITY CLASSIFICATION OF:

a. REPORT unclassified b. ABSTRACT

unclassified c. THIS PAGE

unclassified
17. LIMITATION OF ABSTRACT

UU
18. NUMBER

OF PAGES

4 19a. NAME OF

RESPONSIBLE PERSON 
estimates of both sets of quantities. The SSM thus supports the two main objectives of the GPM-GV: error characterization of the standard retrieval products for data assimilation in realtime weather and hydrometeorological forecasting, and continuous improvement of the GPM standard algorithms via reporting of product bias and uncertainty.

There is a third concept associated with GPM ground validation - the notion of a "Super Site." The working definition for a GV Super Site is simply this: it is an instrumented ground location that executes all of the functions associated with a GV MOV and SSM. Taken together, the MOV, SSM, GV super sites, and supporting systems and services make up the GPM Ground Validation Segment (GVS).

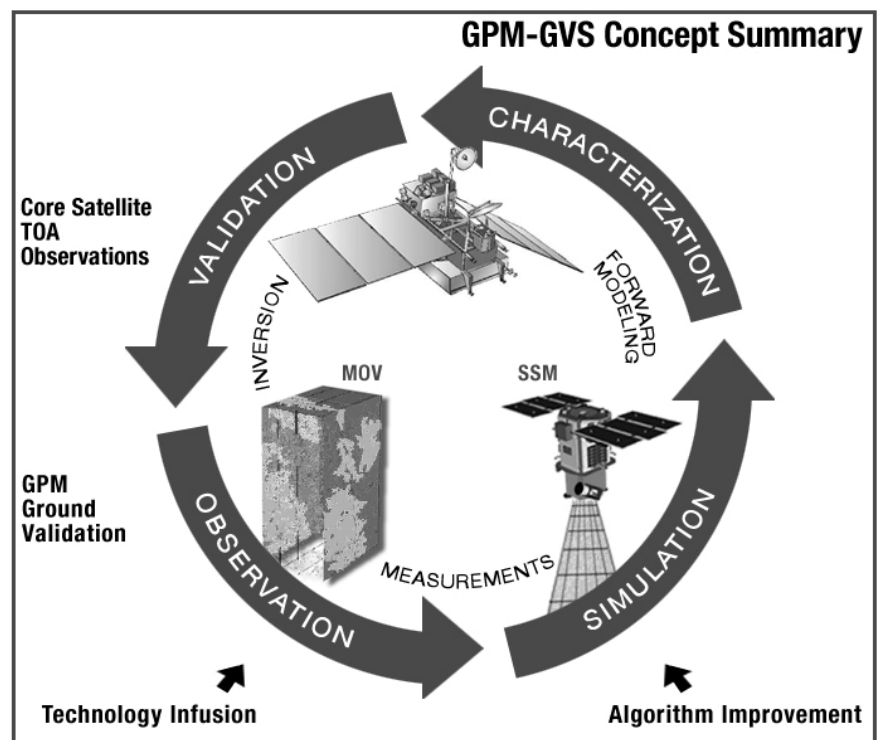

Figure 1. Conceptual framework for the GPM Ground Validation Segment (GVS)

It is understood that the GPM precipitation algorithms will be built on the highly successful TRMM algorithms and thus are expected to produce precipitation estimates at least comparable in accuracy to TRMM products. These forward models, though not perfect, do yield credible and useful precipitation estimates. A key role for the GV is therefore to provide additional atmospheric and microphysical measurements to improve retrieval algorithms by reducing uncertainties and assumptions in these forward models. The conceptual framework for the GPM GV is illustrated in Figure 1.

At present, the GPM GVS is in the early design stage, with preliminary and "critical" (final) design reviews scheduled for February 2006 and June 2007, respectively. The development of the GVS is scheduled to begin after successful completion of these reviews. GVS operations will begin in the year prior to the launch of the GPM core satellite, which has a launch date scheduled for December 2010. The sections below describe three scenarios that are intended to explain how the
GVS will be used during operations, and how its use will meet the goals described in Section I of this paper.

\section{PRe-LAUnCh GRound VALidation ScEnARIO}

The time frame for this scenario is set during GVS pre-launch operations. At this time the GVS routinely collects measurements of atmospheric variables and ground-level precipitation. The atmospheric measurements are used to generate standard products of top-of-the-atmosphere (TOA) TB's and Z's, as well as precipitation estimates based on running the standard retrieval algorithm both constrained with ground measurements and in its normal, unconstrained mode. After several months of observations, but just prior to the launch of the GPM Core Satellite, an algorithm developer on the GPM Precipitation Measuring Mission (PMM) Science Team notes that there appears to be a striking bias in the results of the algorithm run in the constrained and unconstrained mode when estimating of low rainfall rates in the case of convective storms over NASA's oceanic Super Site. He reports this observation during a PMM Science Team meeting. But before taking any action to revise the standard algorithm, the PMM Science Team decides to wait to see if TOA and retrieval results are confirmed in actual measurements of the GPM Core Satellite.

GVS system elements exercised in this scenario:

- Pre-launch measurements by the MOV; estimates of TOA $\mathrm{TB}$ and $\mathrm{Z}$; constrained and unconstrained retrievals

- Reporting from the GVS to the PMM Science Team.

Other features:

- The GPM core satellite is not yet in orbit, but the GVS can simulate satellite observations and algorithm retrieval.

\section{POST-LAUnCH AlgORITHM VALIDATION SCENARIO}

The time frame for this scenario is set during the second year of GPM core satellite operations. An algorithm developer from the PMM Science Team believes that she has an improvement in passive microwave model parameterization that permits better characterization of convective rain over the ocean. She searches the GVS for a representative sample of rain events over the GPM oceanic "super site" and orders 100 precipitation events of interest: 30 had corresponding satellite overpasses, 70 did not. She runs the GPM standard retrieval and her new retrieval algorithm using the GVS-SSMestimated TOA Tb's for the full 100 events in the unconstrained mode, and she also runs the retrieval constrained with GVS-MOV measurements of atmospheric variables. The unconstrained standard algorithm overestimates precipitation in low rain rates (see the previous scenario), but her unconstrained "new" algorithm, her constrained new algorithm, and the constrained standard algorithm all converge on an estimate that compares favorably with GVS-MOV ground measurements of precipitation. The algorithm developer publishes her results. The results are reviewed at a meeting of the PMM Science Team, and the 
team subsequently recommends that her algorithm improvement should be adopted by GPM. She delivers her code and documentation to the GPM Precipitation Processing System (PPS) which integrates the improvement into the standard algorithm. The GVS also integrates the improvements into the SSM forward model. The new forward model is used to generate subsequent GVS products, and to reprocess appropriate products in the GVS archive.

GVS system elements exercised in this scenario:

- GVS search and order

- GVS MOV measures and records precipitation and the atmospheric properties needed by the SSM

- GVS SSM generates products of TOA Tb (and Z)

- GVS interface to the PPS for receipt of algorithm updates.

Other features:

- GPM algorithm improvement is supported by the GVS, but there is no direct requirement on the GVS for conducting algorithm improvement.

- Improved algorithms are integrated into the PPS and GVS following approval by the PMM Science Team.

\section{Global ERror and Bias Determination SCENARIO}

The time-frame for this scenario covers a number of years in the pre- and post-launch GPM era. For the purpose of this scenario, let's assume that a NASA Research Announcement (NRA) is released in 2009 that includes a solicitation for proposals to develop algorithms that estimate global precipitation retrieval errors, bias, and spatial error correlation, with the goal of including these algorithms in the operational PPS.

An atmospheric scientist with a background in modeling, error analysis and assimilation submits a successful proposal to estimate microwave radiometer precipitation errors using GMI, DPR and GVS data. In 2010, the Principal Investigator (PI) places a standing order in the newly operational GVS for all rainfall events over the two NASA GVS sites. Over a period of six months he completes an analysis of the temporal autocorrelation in the GVS measurements of precipitation events, and he begins to collect a statistically independent sample of data.

The PI begins his analysis using GPM constellation data and GVS data to estimate model and retrieval errors for the PPS constellation product. To estimate model errors, he computes the variance in satellite observations of Tb compared to GVS model-based estimates. To estimate retrieval errors, he computes the variance in PPS-computed instantaneous precipitation rate compared to GVS estimates of precipitation rate in the same field of view. This approach only provides error statistics for a small part of the full database of precipitation retrievals used by PPS algorithm.

In 2011, the PI begins work on a global instantaneous precipitation error and bias estimates following the successful launch and checkout of GPM. For this he plans to use the
DPR-derived estimates of atmospheric variables that can be used to parameterize the standard algorithm in much the same way that the algorithm is constrained with ground measurements by the GVS. Indeed, he uses GVS data to validate the fidelity of the DPR-based parameterization, to assess the retrieval results for both DPR-parameterized algorithm compared to the GVS-parameterized algorithm, and to compare both of these results to precipitation actually measured at the GVS super sites.

Satisfied with the results so far, he enhances his global error algorithm by accumulating statistics for the difference between the [Standard-Algorithm(constrained by DPR) Standard-Algorithm(unconstrained)] for each bin of $\mathrm{Tb}$ ensembles in the standard algorithm database. He publishes a paper on algorithm bias, and as 2012 draws to a close he plans to use the database as the foundation for a grant renewal focused on estimating global error covariance in rainfall retrievals.

GVS system elements exercised in this scenario:

- GVS search, order, standing order

- GVS MOV measures and records precipitation and the atmospheric properties needed by the SSM

- GVS SSM generates products of TOA Tb, Z, and constrained precipitation retrievals

- GVS interface to PPS for receiving PPS product subsets.

Other features:

- Global error estimation is supported by the GVS, but there is no direct requirement on the GVS for making global error estimates (it is assumed that global error products will be generated by the PPS based on algorithms provided by the PMM Science Team).

\section{CONClusions AND NeXt StePs}

At present, the GPM GVS is in the early stage of design. The set of objectives described in Section I of this paper are intended to provide the guiding direction as the GVS progresses through design and into development. Additionally, the MOV and SSM described in Section II provide a conceptual framework for achieving GPM GV objectives using measurements made with ground-level instrumentation (via the MOV), and model-based analysis (via the SSM).

The set of scenarios presented earlier illustrate how the GVS MOV and SSM will be used during the GPM era. These scenarios are useful for two purposes. First, they provide a check, verifying that the objectives of the GPM ground validation can be met with the "end state" version of GVS. Secondly, the scenarios are a point of departure for system engineering, particularly in the development of a GVS functional architecture, requirements, and operations concept.

A comparison of the scenarios to the GPM ground validation objectives listed in Section I shows a relatively good match-up between the two. 
Scenarios 1 and 2 address the quantitative uncertainties in the standard precipitation retrieval algorithms (Goal 1) when comparing ground-based estimates of precipitation to precipitation estimates generated by standard GPM algorithms. This is essentially the standard approach to validation: comparing satellite retrievals to "ground truth" and evaluating the differences. Although this is valuable approach, it has two major limitations for the GPM mission. The first limitation is related to the problem of sample size. The joint probability of a precipitation event at a GVS Super Site coinciding the field of view of a GPM satellite overpass is relatively low, and this is especially true for the DPR (swath width of about $245 \mathrm{~km}$ for the Ku band and about $100 \mathrm{~km}$ for the Ka band in the across-track direction; Satoh et al., 2003). The ground truth approach to validation may result in unacceptably small sample sizes of coincident precipitationoverpass events even after a period of 3 years, the nominal lifetime of the GPM core satellite. The second problem with this approach is one of algorithm degeneracy. Many combinations of TOA Tb's or Z's can map to a single precipitation rate in the GPM standard algorithms. Consequently, a comparison of the algorithm retrieval to ground truth may reveal that they are the same (or different), but simple comparisons will not help explain how or why those similarities (or differences) exist.

Scenarios 1 and 2 demonstrate how the GVS can contribute to the improvement of GPM precipitation retrieval algorithms (Goal 2). These scenarios show how the MOV-SSM combination utilizes a forward model to simulate TOA observations collected by the DPR and GMI instruments on the GPM satellite. These simulated observations will be compared to actual satellite measurements. This approach avoids the degeneracy problem noted above because any source of error in the comparison can be traced to errors in the ground-based instrumentation, to the forward model, or (however unlikely) to error or bias in the GPM instruments. The model-based approach to validation also ameliorates the sample size problem because the forward model can be run for any precipitation event, whether the satellite is in view or not. The criterion for validating the radiative transfer models that serve as the basis for the GPM precipitation retrieval algorithms thus becomes a comparison of GVS model-based estimates with satellite TOA observations. If they are identical to within some noise level then we will have confidence that they are accurate for the range of atmospheric conditions observed by the MOV. If they are different, then either the MOV measurements or the SSM model is in error. The errors identified by the GVS will be used over the mission lifetime in a series of successive improvements to the forward model, to the parameterization of the forward model, to the GPM standard retrieval algorithms, and to the ground instrumentation.

Finally, as illustrated by scenario 3, the GVS can contribute to understanding the space and time error characteristics of GPM precipitation products generated by the GPM standard algorithms (Goal 3). Clearly, the GVS observations will be limited to the relatively small geographic area of NASA's continental and oceanic Super Sites. Consequently, the ability of the GVS to provide error and bias estimates will also be limited to its small geographic regime. Generalizing from local to global error estimates will require that the key GVS parameters have some counterpart (proxy) that can be observed globally, so that the true variability can be estimated with some confidence. In the GPM era, it is expected that such global proxies may be retrieved from measurements made by the Dual-frequency Precipitation Radar (DPR), and perhaps other instruments. However, no methods currently exist for generalizing local GVS error estimates to a large regional or even global scale. Thus, it is expected that global error characterization will be conducted as a research activity that is beyond the scope of the GVS. Once such research is mature, it is expected that global error products will be generated by the Precipitation Processing System (PPS) based on algorithms provided by the science community. The GVS will provide error reports to the PPS as input for the generation of GPM global error products.

Because NASA's GVS is in an early design stage, the feasibility of actually implementing the GPM GVS according to the conceptual framework presented here has not yet been demonstrated. To address this issue, a team was selected by peer-review to study the feasibility of the MOV and SSM concepts identified above, and to contribute to the final design of NASA's GVS. The members selected for this team include:

\section{Chandrasekar, Colorado State University \\ Robert Meneghini, NASA/GSFC \\ William Olson, NASA/GSFC \\ Steven Rutledge, Colorado State University (team leader for a consortium that includes additional members from other institutions) \\ Sandra Yuter, University of Washington}

Reports from the GPM Ground Validation feasibility studies are expected in late summer or early fall of 2005 . These results, along with a planned prototype activity, will contribute to the design of NASA's GPM Ground Validation Segment. A preliminary GVS design is expected to be complete in February 2006. The final GVS design is expected to be complete by July 2007. Following this, instrumentation will be fielded, analysis capabilities established, and the GVS will be operational in the year prior to the launch of the GPM core satellite, which has a launch date scheduled for December 2010 .

\section{REFERENCES}

Flaming, G.M., "Measurement of Global Precipitation," Proceedings of the International Geoscience and Remote Sensing Symposium, Anchorage, Alaska, September 20-25, 2004.

Satoh, S., R. Oki, N. Takahashi, T. Iguchi, "Development of spaceborne dual-frequency precipitation radar and its role in the global precipitation measurement," EGS-AGU-EGU Joint Assembly, Nice, France, 6-11 April, 2003 (abstract). 